\title{
Development of paper-based color test-strip for drug detection in aquatic environment: Application to oxytetracycline
}

\author{
Helena I.A.S. Gomes, M. Goreti F. Sales
}

\begin{abstract}

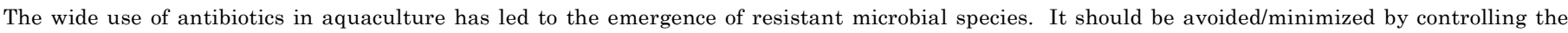

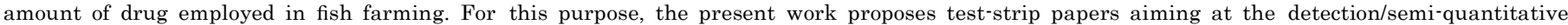

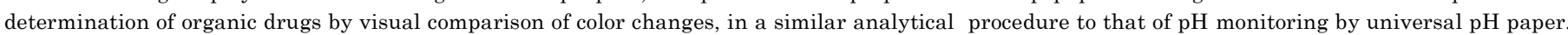

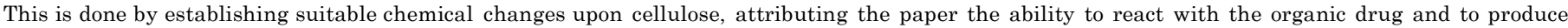

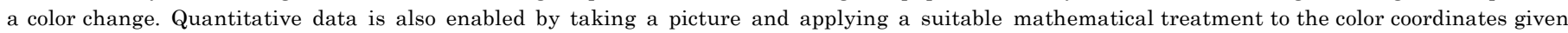
by the HSL system used by windows.

As proof of concept, this approach was applied to oxytetracycline (OXY), one of the antibiotics frequently used in aquaculture. A bottom-up

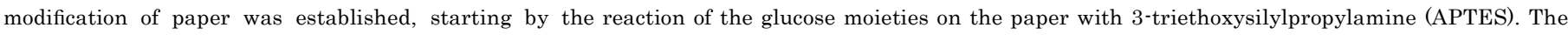

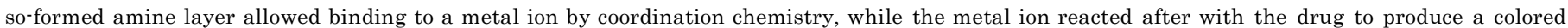

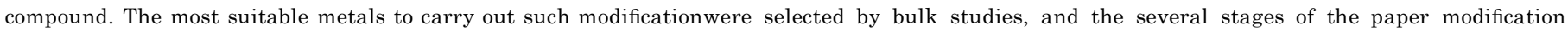

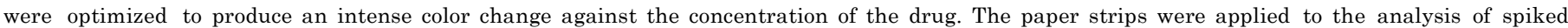

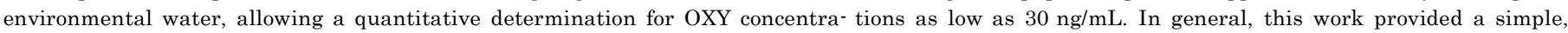
method to screen and discriminate tetracycline drugs, in aquaculture, being a promising tool for local, quick and cheap monitoring of drugs.
\end{abstract}

Keywords

Test-strip, Paper-based, Antibiotic, Water, Environment, Aquaculture

\section{Introduction}

Water is a renewable but finite resource. By 2030, global demands of water will exceed more than $40 \%$ the existing resources and more than a third of the world's population will have to deal with water shortages (European Environment Agency (EEA) 2010). Efforts are currently being made throughout Europe towards a reduced and efficient water use and prevention of any further deterioration of the quality of water (European Commission, 2010; European Environment Agency, 2010; European Parliament and of the Council, 2006).

Pharmaceutical drugs are currently among the most common and dangerous organic contaminants, playing a great impact upon the quality of water and biodiversity. Despite its great importance for meeting the needs of the world fish supplies, the aquaculture sector may easily lead to the introduction of drugs in the environment, most importantly in water. A wide range of drugs are employed in intensive fish farming activities, being the use of antibiotics of great concern to public health and environment. The most important classes and groups of antibiotics used in aquaculture include penicillins, cephalosporins, tetracyclines, aminoglycosides, macrolides, glycopeptides, sulfonamides, and quinolones (Kuemmerer, 2009).

Antibiotics are added directly to the water rendering high local concentrations both in water and in adjoining sediments (Cabello, 2006), contributing to increase the total concentration of these drugs in the water and disseminate these throughout the environment (Kuemmerer, 2009). There are three main risks deriving from the presence of antibiotics in the waters: direct organic damage to water; impact on the biotic environment; and lastly, the most feared one, indirect effects on health via resistant microorganisms (Kemper, 2008; Li et al., 2012; Luis Martinez, 2009; Sarmah et al., 2006). The increasing emergence of antibiotic resistance in human pathogens is today a great concern to public health, not only for hindering the successful treatment of infectious diseases, but also for obstructing the combat to other 

pathologies in which antibiotic prophylaxis is needed for avoiding associated infections (Luis Martinez, 2009; World Health Organization, 2000).

Avoiding the environmental impact of antibiotics promoted by fish farming activities is thus fundamental. Considering that antibiotics are essential to maintain fish in healthy conditions for long periods of time under intensive production, the introduction of antibiotics upon the aquatic environment should be set to a minimum. This (apparently) simple target requires a simple measuring process, to enable drug control in multiple stages, after the drug is introduced in water. A test strip material, equipmentfree, would meet these requirements.

As far as we know, there are only two colored sensing strips available in the literature for detecting drugs in aquatic environment, both devoted to Quinolone antibiotics (Lara Guerreiro and Ferreira Sales, 2011; Silva et al., 2012). These strips consisted of plasticized PVC entrapping a suitable reagent, with which the antibiotic would form a colored complex. The colorimetric reaction took place in the solid/liquid interface established between the plasticized PVC layer (containing the reagent) and the sample solution (containing the antibiotic). Although the analytical procedure was simple and successful, there are however two aspects of the conception of these strips that could be improved: (1) the supporting material of the strip should consist of renewable material to diminish the environmental impact of an intensive use of such plasticized PVC strips; (2) the complexing reagent should be immobilized in an organized nanostructured assembly (instead of simple entrapment) to allow lowering the detection limit of the test-strip.

Thus, this work proposes the use of cellulose paper as supporting and its subsequent chemical modification by a self-assembly approach to bind a reagent that would sensitize this material to the presence of the antibiotic. This concept was applied herein to Oxitetracycline (OXY), an antibiotic of the tetracycline group. Since the detection/determination of the antibiotic should account a color change to avoid the use of analytical equipment, OXY was made react first with different metals, identifying this way the most suitable colored complex in this context. The metal(s) was then attached to the cellulose paper following suitable nanostructured modification. The optimization and characterization of the final paper test-strip is described, along with the application of the final sensory material. In all cases, the detection/determination of OXY is assessed by visual detection of the color change or mathematical handling of the color coordinates of the images, captured by a normal camera and read in Paint software of Windows.

\section{Experimental section}

\subsection{Materials and reagents}

Deionised water o $0.1 \mu \mathrm{S} / \mathrm{cm}$ was employed throughout this work. Chemicals were pro-analysis grade and obtained from different sources: iron (III) chloride $\left(\mathrm{FeCl}_{3}\right)$ 6-hydrate (Scharlau), lead (II) nitrate (Riedel Haen), copper (II) sulfate 5-hydrate (Panreac), ammonium chloride (Panreac), hydrochloric acid $(\mathrm{HCl}$, Panreac), sodium hydroxide ( $\mathrm{NaOH}$, Scharlau), calcium hydroxide (Panreac), magnesium chloride 6-hydrate (Panreac), aluminum sulfate 16-hydrate (BDH), 4-(2-hydroxyethyl)-1-piperazineethanesulphonic acid (HEPES, Sigma), tetracycline (Applichem), zinc chloride (Merck), ethylenediamine (Merck), glutaraldehyde (GLUT, Fluka), chloramphenicol (CRP, Fluka), oxytetracycline (OXY, Fluka), sulfadiazine (SDZ, Fluka), norfloxacin (NOR, Fluka), amonium chloride $\left(\mathrm{NH}_{4}\right.$, Merck), disodium hydrogenephosphate $\left(\mathrm{PO}_{4}{ }^{3-}\right.$, Sigma Aldrich)and 3-triethoxysilylpropylamine (APTES, Acros).
The ethanol used was of $96 \%$ and the paper for the test strip was from Fanoia (Reference S-300).

\subsection{Apparatus}

Spectrophotometric measurements were made in a Thermo Scientific Evolution 201, spectrophotometer. The digital image of the paper test-strips was acquired by a digital camera Samsung PL150, 8 megapixels. The color coordinates of each image was acquired by the HSL color system (Hue, Saturation and Lightness of the HSL space) and measured by using the Paint program of Windows. An ultrasonic bath (Bandelin Sonorex Digitec, model DT 31) and/or a magnetic stirrer (Scansci, MS-H280Pro), were used to promote the dissolution of the solids.

Infrared spectra were collected at a Fourier Transformed Infrared Spectrometer (FTIR, Thermo Scientific Nicolet IS10) equipped with an Attenuated Total Reflectance (ATR) accessory of diamond crystal.

\subsection{Metal selection}

The colorimetric reaction was tested between OXY and Fe(III), $\mathrm{Al}(\mathrm{III}), \mathrm{Pb}(\mathrm{II}), \mathrm{Mg}(\mathrm{II}), \mathrm{Cu}(\mathrm{II}), \mathrm{Ca}(\mathrm{II}), \mathrm{NH}_{4}{ }^{\mathrm{p}}$ e $\mathrm{Zn}(\mathrm{II})$. This was done by mixing $2.0 \mathrm{~mL}$ OXY solution $1.0 \times 10^{-3} \mathrm{~mol} / \mathrm{L}$ with $1.0 \mathrm{~mL}$ of an aqueous solution contained the metal species with a concentration of $1.0 \times 10^{-2} \mathrm{~mol} / \mathrm{L}$. Acidic and neutral conditions were tested by preparing all solutions in $1.0 \times 10^{-3} \mathrm{~mol} / \mathrm{L} \mathrm{HCl}$ or $1.0 \times 10^{-2} \mathrm{~mol} / \mathrm{L}$ HEPES. When in the presence of color, the UV/Vis spectrum was recorded to assess the wavelength maximum absorbance and the corresponding absorption intensity.

\subsection{Chemical modification of cellulose}

The cellulose paper was cut in $1 \times 1 \mathrm{~cm}^{2}$ peaces and chemically modified by incubating these pieces in different solutions/conditions. The incubation was always conducted under constant stirring (lateral motion), usually at $20^{\circ} \mathrm{C}$ (unless specified otherwise), protected from light and ensuring that the paper was completely submerged in the solution. This modification followed the three different stages presented next and was followed by Raman spectroscopy studies (the samples were analyzed directly as modified, not requiring previous sample pre-treating).

\subsubsection{Reaction with APTES}

The first modification of the paper aimed its amination and was tried out in different ways: (i) incubating the paper in APTES solution prepared in ethanol, in different concentrations $(1 \%, 10 \%$, $50 \%$ and $100 \%$ ), and for up to $3 \mathrm{~h}$; (ii) incubating the paper in sodium periodate solution $(0.20 \mathrm{~mol} / \mathrm{L}$ in water, adjust to $\mathrm{pH} 4.4$ by adding few drops of $\mathrm{NaOH} 1.0 \mathrm{~mol} / \mathrm{L}$ ) for up to $2 \mathrm{~h}$, proceeded by a second incubation in ethylenediamine solution ( 2 mol ethylenediamine solution: 1 mol sodium periodate solution); (iii) or incubating the paper in potassium persulphate $0.20 \mathrm{~mol} / \mathrm{L}$, added of sodium hydroxide $1.0 \mathrm{~mol} / \mathrm{L}$ until the $\mathrm{pH} 4.4$ was reached, and followed by incubation in ethylenediamine solution, in a similar approach to that indicated previously. The success of these different modifications was followed by completing the modification and incubating, for $5 \mathrm{~min}$, the modified paper in aqueous solutions of OXY with different concentrations $\left(1.0 \times 10^{-2} \mathrm{~mol} / \mathrm{L}\right.$, $5.0 \times 10^{-3} \mathrm{~mol} / \mathrm{L}$ and $1.0 \times 10^{-3} \mathrm{~mol} / \mathrm{L}$ ).

\subsubsection{Selection of metal concentration}

The cellulose/APTES material was after incubated in solutions of the selected metal of $1.0 \times 10^{-3}, 1.0 \times 10^{-2}$ and $1.0 \times 10^{-1} \mathrm{~mol} / \mathrm{L}$. The produced cellulose/APTES/Metal material 
was incubated next in OXY solution of $1.0 \times 10^{-2} \mathrm{~mol} / \mathrm{L}$, for $5 \mathrm{~min}$, and the results were evaluated by visual comparison.

\subsubsection{APTES reaction time}

The time given for the amination reaction was thought relevant and optimized next. For this purpose, the time given for the reaction between cellulose and APTES was varied from 2 to $30 \mathrm{~min}$ of reaction (checking the color change each $2 \mathrm{~min}$ ) and then for 1, 2 and $3 \mathrm{~h}$. The OXY concentration used in this test was $1.0 \times 10^{-5} \mathrm{~mol} / \mathrm{L}$.

\subsection{OXY assays in the paper test strip}

The response of the test strip to OXY was checked by submerging for $5 \mathrm{~min}$ the cellulose/APTES/Metal material in standard aqueous solution of the antibiotic. The paper was then removed and let dry at ambient temperature. The picture of the paper was taken without specific requirement and the color coordinates obtained by analyzing the image in the Paint program. The HSL color system was selected and at least three different points per paper were considered.

The procedure was applied to characterize the calibration features of the paper test strip, its selectivity and its feasibility of application to the analysis of environmental samples. The calibration was set for aqueous solutions of antibiotic with 14 different concentrations, varying from $5.0 \times 10^{-9}$ to $1.0 \times 10^{-2} \mathrm{~mol} / \mathrm{L}$. The cross-response of the paper test-strip to other antibiotics was tested for norfloxacin, sulfadiazine, chloramphenicol and tetracycline, regularly used in aquaculture activities. The concentration of these drugs was set to $1.0 \times 10^{-2} \mathrm{~mol} / \mathrm{L}$. Considering that the major components linked to wastewater discharges in aquatic environment systems are P and N (Colt, 2006), phosphate and ammonium were tested as possible interfering species. Their concentrations were set to $4.0 \times 10^{-6}$ and $1.0 \times 10^{-4} \mathrm{~mol} / \mathrm{L}$, respectively, the maximum value allowed for environmental discharge (Decreto-Lei no. 236/98). The use of the test strip at the quantification of OXY in environmental waters was tested by spiking such waters with $1.0 \times 10^{-3} \mathrm{~mol} / \mathrm{L}$, collecting the color coordinates of the incubated papers and calculating the corresponding concentration by using the calibration data.

\section{Results and discussion}

\subsection{Colored complex}

The visual detection of the antibiotic drug in water implicated its chemical change into a colored compound on the surface of the paper test-strip. Since metal species are used in many occasions to form colored complexes with organic compounds, the reaction of OXY with a metal was pondered. This reaction should be sensitive enough to allow low detection limits while providing significant color change for conditions with/without OXY.

The selection of a suitable metal to be used as colored complexing agent for OXY was made by trial and error approach. A batch of possible ionic species was tested for this purpose: $\mathrm{Fe}(\mathrm{III}), \mathrm{Al}(\mathrm{III}), \mathrm{Pb}$ (II), $\mathrm{Mg}$ (II), $\mathrm{Cu}$ (II), $\mathrm{Ca}(\mathrm{II})$, and $\mathrm{Zn}$ (II). Each species was tested in both acidic and neutral conditions, because the presence of color and its intensity are linked to the $\mathrm{pH}$ of the solution. Alkaline conditions were not evaluated herein due to occurrence of highly insoluble hydroxides metal derivatives. The concentrations of metal and drug were set to high values, to $1.0 \times 10^{-3} \mathrm{~mol} / \mathrm{L}$, ensuring that the results would be relevant and independent form the limit of detection.

From all inorganic species tested, only $\mathrm{Fe}$ (III) and $\mathrm{Cu}$ (II) were capable of addressing the expected targets. The blue color of the copper solution changed to green when OXY was present, while the yellow light color of the iron solution changed to orange with OXY. Blank controls and tests with individual solutions were made at different $\mathrm{pHs}$ to confirm that the previous color changes resulted from the reaction between the metal and the drug.

\subsection{Amination of cellulose paper}

Both $\mathrm{Fe}$ (III) and $\mathrm{Cu}$ (II) are able to bind to amine groups. According to the literature, copper(II) complexes with amine often accommodate four ligands leading to the formation of the diaquatetramminecopper(II) ion/tetraamminecopper(II) ion (Brown, 2000-2013). This is presented as a ligand-exchange reaction where no redox change of copper exists. The coordination number of copper remains at 6 and the overall charge of the complex remains at $p 2$ because ligands are electrically neutral. Similar considerations could be established for iron, binding to amine and

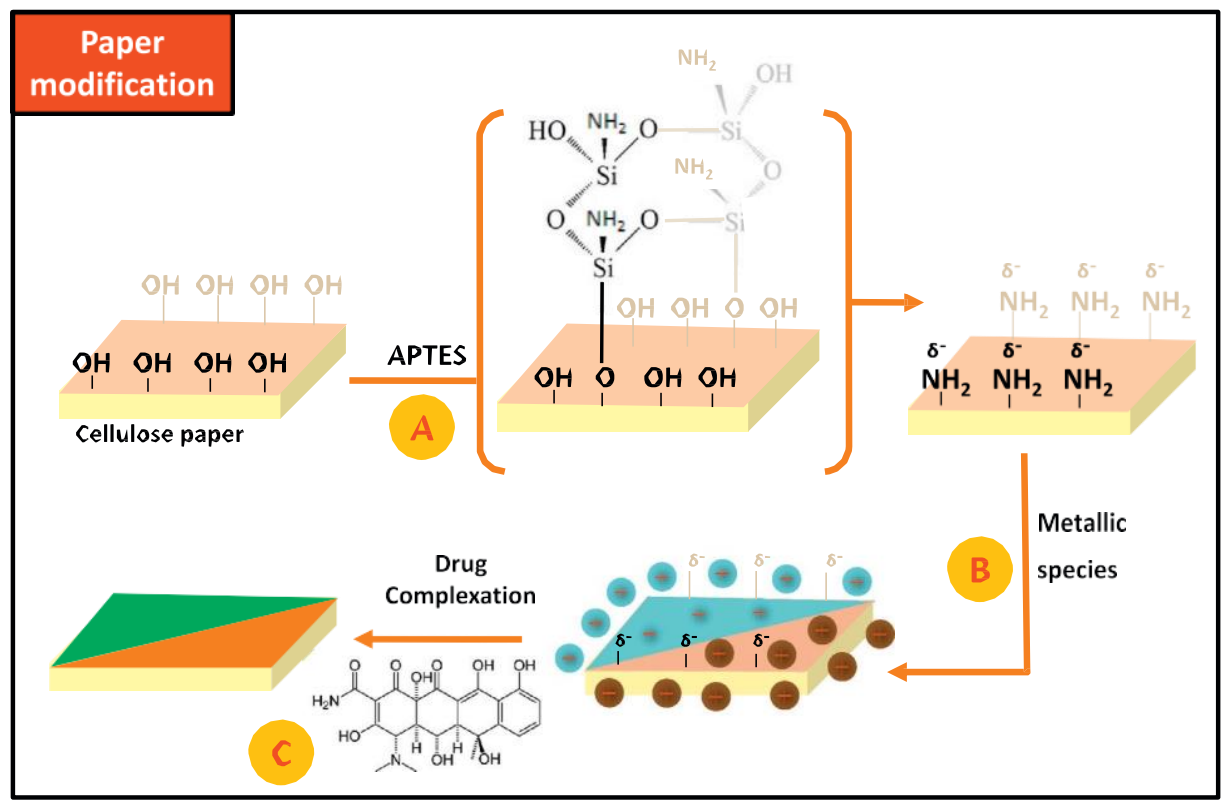

Fig. 1. The different stages of chemical modification of the paper. 
leading to no redox change at the iron core element. Thus, the next stage of the paper modification concerned its transformation into an amine surface (Fig. 1).

Two distinct approaches were taken to change the cellulose matrix of the paper: direct reaction of the paper with an aminebased reagent or oxidation of the cellulose for subsequent binding of an amine compound. The later process is simpler but requires adjacent hydroxyl groups in the cellulose for an effective binding of the silane reagent, while the former is more complex but involves conventional and successful chemistry (the oxidation of hydroxyl to carboxylic groups, their activation by EDAC/NHS chemistry and subsequent amine binding). The later made use of APTES reagent while the former was tried out by using periodate or persulfate oxidizing agents.

Color changes were observed only when APTES was used. The results recorded at this staged were obtained with a solution of $10 \%$ APTES, allowed to react for $3 \mathrm{~h}$, and a constant copper/iron concentration, set to $1.0 \times 10^{-2} \mathrm{~mol} / \mathrm{L}$ (in this and in all other first assays). As may be seen in Fig. 2A, the modified paper showed a blue color when in contact with a blank solution and a green color when in contact with the drug solution. The intensity of the green color increased with the concentration of the drug, allowing a visual perception of the semi-quantitative amount present in the solution.

All chemical modifications made to the paper were followed by Raman spectroscopy (Fig. 3). The cellulose paper use in this study shows skeletal bending modes of $\mathrm{C}-\mathrm{C}-\mathrm{C}, \mathrm{C}-\mathrm{O}-\mathrm{C}, \mathrm{O}-\mathrm{C}-\mathrm{C}, \mathrm{O}-\mathrm{C}-\mathrm{O}$ in the $150-550 \mathrm{~cm}^{-1}$ region (Szymanska-Chargot et al., 2011). In general, the region between 800 and $1180 \mathrm{~cm}^{-1}$ is dominated by $\mathrm{C}-\mathrm{C}$ and $\mathrm{C}-\mathrm{O}$ stretching motions and some $\mathrm{H}-\mathrm{C}-\mathrm{C}$ and $\mathrm{H}-\mathrm{C}-\mathrm{O}$ bending. It is further expected that the region of $1180-1270 \mathrm{~cm}^{-1}$ is assigned to bending of $\mathrm{H}-\mathrm{C}-\mathrm{C}, \mathrm{H}-\mathrm{C}-\mathrm{O}, \mathrm{H}-\mathrm{C}-\mathrm{H}$ and $\mathrm{C}-\mathrm{O}-\mathrm{H}$; $1270-1350 \mathrm{~cm}^{-1}$ to bending of $\mathrm{H}-\mathrm{C}-\mathrm{C}$ and $\mathrm{H}-\mathrm{C}-\mathrm{O}$; and

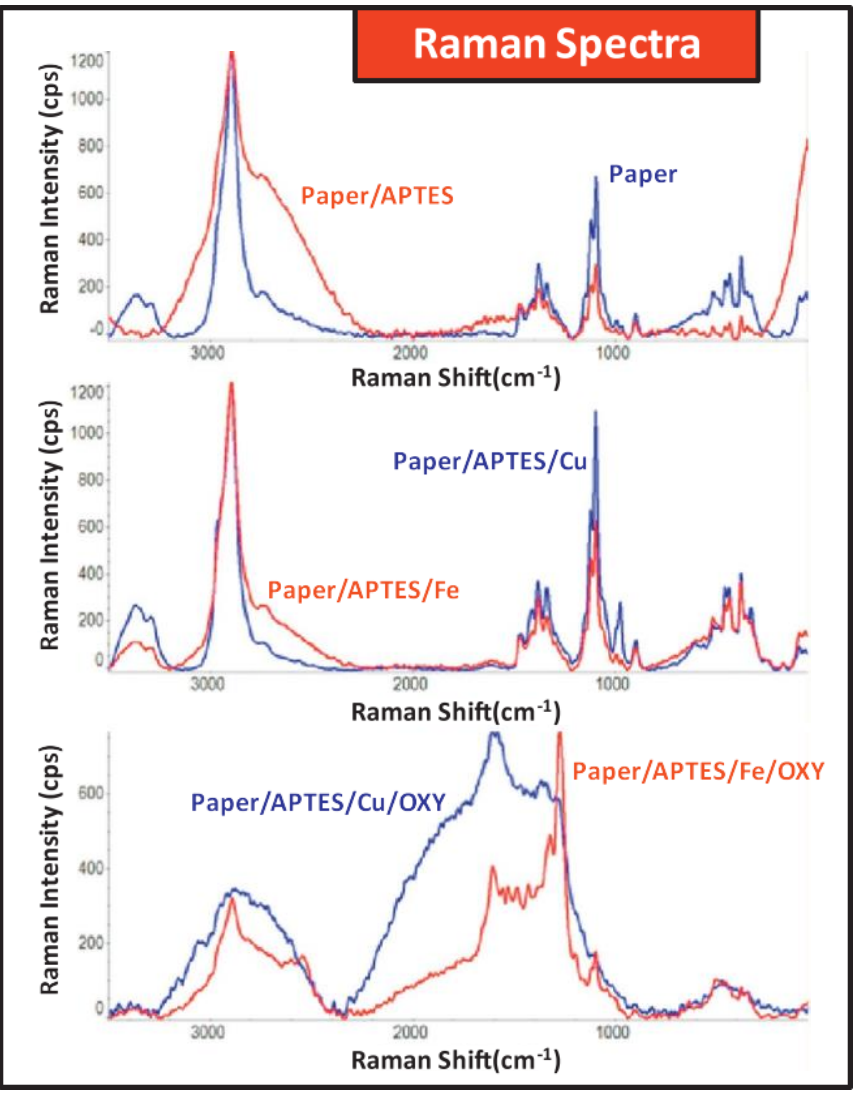

Fig. 3. RAMAN spectra of the different stages of the paper test strips.

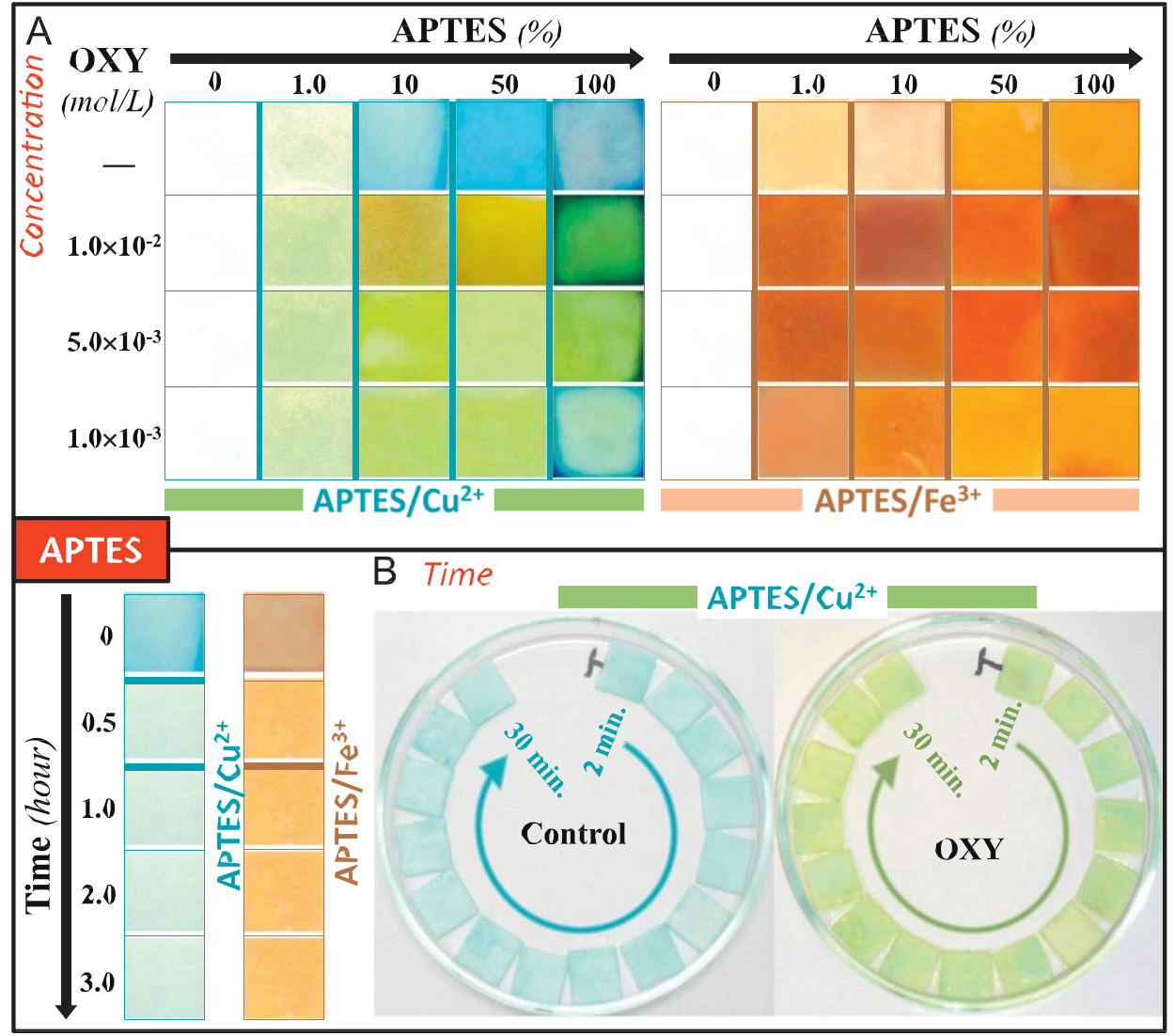

Fig. 2. The effect of changing the concentration (A) and the time (B) given for the reaction with APTES. 
$1350-1430 \mathrm{~cm}^{-1}$ to bending of $\mathrm{C}-\mathrm{O}-\mathrm{H}$. Above $2500 \mathrm{~cm}^{-1}$ two bands occur: a very intensive one at $2900 \mathrm{~cm}^{-1}$, which indicates mostly $\mathrm{C}-\mathrm{H}$ stretching vibrations and a very broad one between 3200 and $3500 \mathrm{~cm}^{-1}$, corresponding to $\mathrm{O}-\mathrm{H}$ stretching vibrations (Szymanska-Chargot et al., 2011).

As may be seen in Fig. 3, the intrinsic spectra of the cellulose surface used in this study changed after binding APTES (to spectra). The major alterations were located within $320-1500 \mathrm{~cm}^{-1}$ and $3180-3500 \mathrm{~cm}^{-1}$ and the $2895.29 \mathrm{~cm}^{-1}$ peak of cellulose shifted to $2913.17 \mathrm{~cm}^{-1}$. The addition of metal (Fe or $\mathrm{Cu})$ to the paper/APTES surface did not change much the Raman spectra, but the observed color was sufficient to confirm their presence (blue for copper and brown for iron). The subsequent addition of OXY to paper/APTES/Fe or paper/APTES/Cu altered significantly the Raman spectra of the material. Overall, strong fluorescence was observed in this, with groups of medium to strong bands within $1160 \mathrm{~cm}^{-1}$ and $1700 \mathrm{~cm}^{-1}$. In addition, the peaks at about $2900 \mathrm{~cm}^{-1}$ and $1150 \mathrm{~cm}^{-1}$ decrease their intensity when OXY was present.

\subsection{Optimization of the test strip design}

The assembly of both paper/APTES/Cu and paper/APTES/Fe materials was optimized for several parameters to increase the sensitivity of the paper-test strip to the presence of OXY. In practice, this principle would be achieved by intensifying the color variation between similar concentrations of OXY. The results were recorded by digital photography and described next.

\subsubsection{APTES concentration}

The concentration of APTES was tested for $0 \%, 1.0 \%, 10 \%, 50 \%$ and $100 \%$ and the time given for this reaction to take place was the same constant. A period of $3 \mathrm{~h}$ was given, to ensure that the reaction occurred in a high extension. Three different concentrations of OXY were tested for each condition, along with a blank test (where no OXY was present).

The results obtained are depicted in Fig. 2A. The presence of APTES was essential in $\mathrm{Cu}$ and Fe paper test strips. No color was observed for papers without APTES, neither after binding the metal nor after reaction with OXY. Indeed, the amount of metal bond to the paper/amine material increased with the increasing concentrations of APTES. This was evidenced by the more intense colors observed for papers exposed to the blank solution. After submerging the papers in solutions of OXY a significant color change was observed: from blue to green for paper/APTES/Cu material and from yellow to orange in paper/APTES/Fe test strip. This color variation depended on the concentration of OXY present, because a gradient could be observed for the different concentrations tested under the paper test strips.
The more intense color gradients were observed for papers modified with $10 \%$ or $50 \%$ concentration of APTES, when considering the color variation within the different concentrations of drug tested. But comparing the color difference between the blank and the lowest OXY concentration indicated that APTES 10\% was more effective, especially in the paper/APTES/Fe test strip (where 50\% APTES gave similar color between blank and $\left.1.0 \times 10^{-3} \mathrm{~mol} / \mathrm{L}\right)$. So, the concentration of APTES set to subsequent studies was $10 \%$.

\subsubsection{APTES reaction time}

Considering the importance of the presence of APTES, the time given for the reaction to take place could be quite relevant. Overall, it was important to identify for how long it was necessary to proceed with the APTES reaction until its maximum extent was reached. A limiting concentration of OXY was used at this stage, equal to $1.0 \times 10^{-5} \mathrm{~mol} / \mathrm{L}$, in order to promote the evidence of subtle differences in the extent of the reaction.

The first tests were conducted for 30 min until a maximum period of $3 \mathrm{~h}$. As may be seen in Fig. 2B (left), no change was observed for the different timing periods, suggesting that the APTES reaction was almost immediate. Then, further studies have been carried out in each $2 \mathrm{~min}$, up to a maximum period of $30 \mathrm{~min}$. The pictures taken to all papers (together and soaked) may be seen in Fig. 2A (right) and evidenced no changes in the color along time.

In general, the studies of APTES reaction within time indicated that the reaction was immediate, suggesting that the assembly of the paper test strip could be a very simple and fast process.

\subsubsection{Selection of metal concentration}

The concentration of the metal species could affect the amount of metal on the test strip that could bind to OXY. Different concentrations of metal species were tested for this purpose, $1.0 \times 10^{-3}, 1.0 \times 10^{-2}$, and $1.0 \times 10^{-1} \mathrm{~mol} / \mathrm{L}$. The tests were conducted in paper with or without OXY, setting the OXY concentration (when present) to $1.0 \times 10^{-2} \mathrm{~mol} / \mathrm{L}$. The results obtained in this study are indicated in Fig. 4.

In general, the higher the concentration of metal in solution, the more intense the color of the blank paper, signaling the presence of more metal ionic species bound to APTES. The color of the paper with OXY solution was also more intense for higher concentrations, leading to more significant differences between to blank (water) and drug solutions. The color observed was almost immediate, and did not change within time, up to a maximum of several months.

Overall, the materials paper/APTES/Cu or paper/APTES/Fe employed as paper test strips were assembled by reacting the blank paper with APTES, $10 \%$, for $2 \mathrm{~min}$, and incubating next the paper/ APTES in $\mathrm{Cu}^{2 \mathrm{~b}}$ or $\mathrm{Fe}^{3 \mathrm{p}}$ solution, respectively, in $1.0 \times 10^{-1} \mathrm{~mol} / \mathrm{L}$, for $30 \mathrm{~min}$.

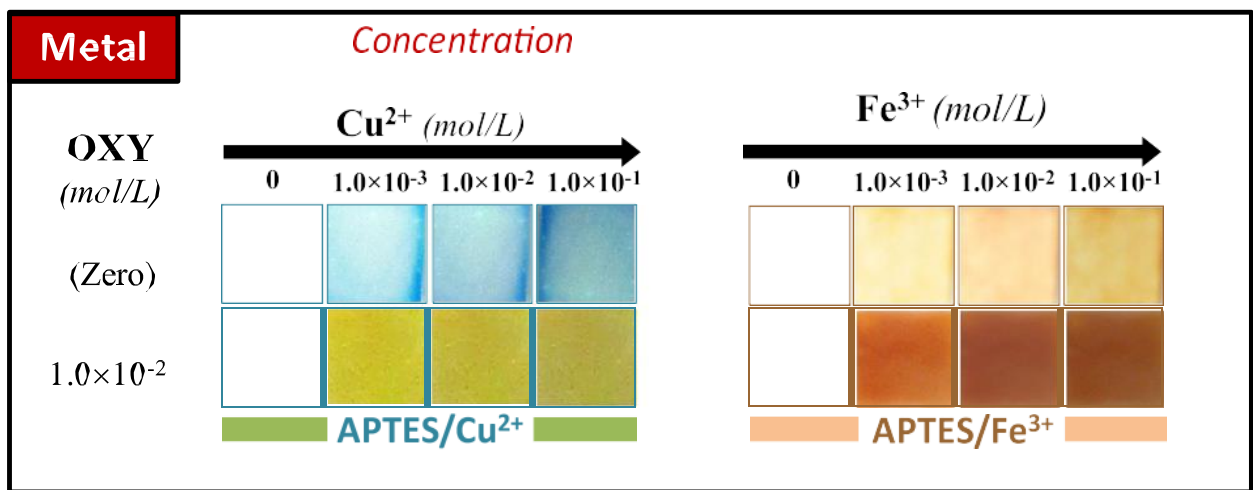

Fig. 4. Effect of metal concentration. 


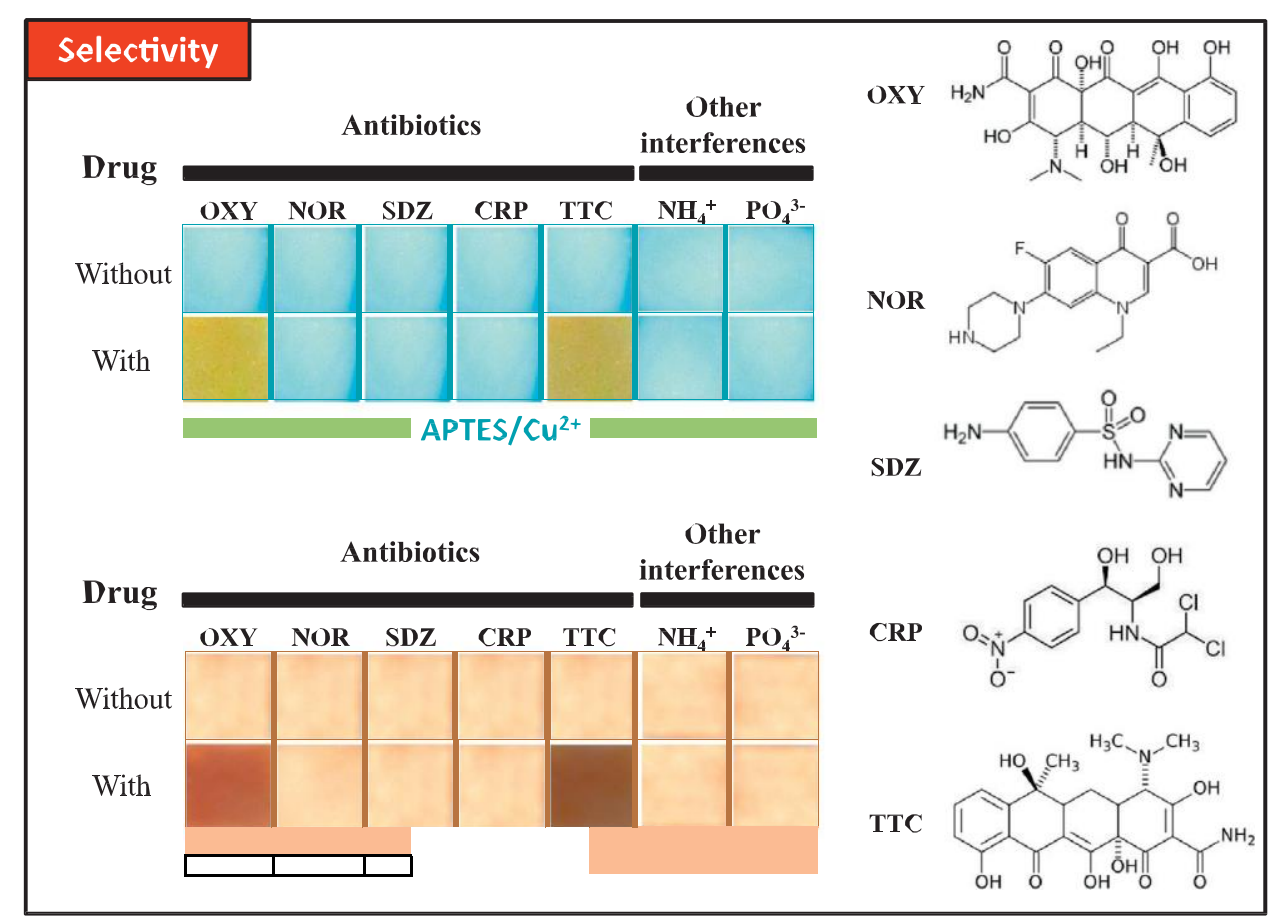

Fig. 5. Cross-response of the paper test strips to other interferences.

\subsection{Cross-response to other compounds}

The selectivity of a method is a key feature when this material is expected to be applied under near-real conditions. Considering the possible use of different antibiotics and other compounds present in aquaculture facilities, the response/cross-response of the paper test-strips to these interferences was checked. This study was carried by incubating the paper test-strips in solutions of OXY, NOR, SDZ, CRP, TTC, $\mathrm{NH}_{4}{ }^{\mathrm{p}}$ and $\mathrm{PO}_{4}{ }^{3-}$. The results obtained are depicted in Fig. 5.

In general, the response of the paper test-strip was not affected by other interferences, except TTC. This observation was independent of the metal present in the sensory paper. Considering that TTC and OXY belong to the same chemical group of antibiotics, it was reasonable to assume that the color changed in result of a reaction to a common nucleus structure among Tetracycline's. This

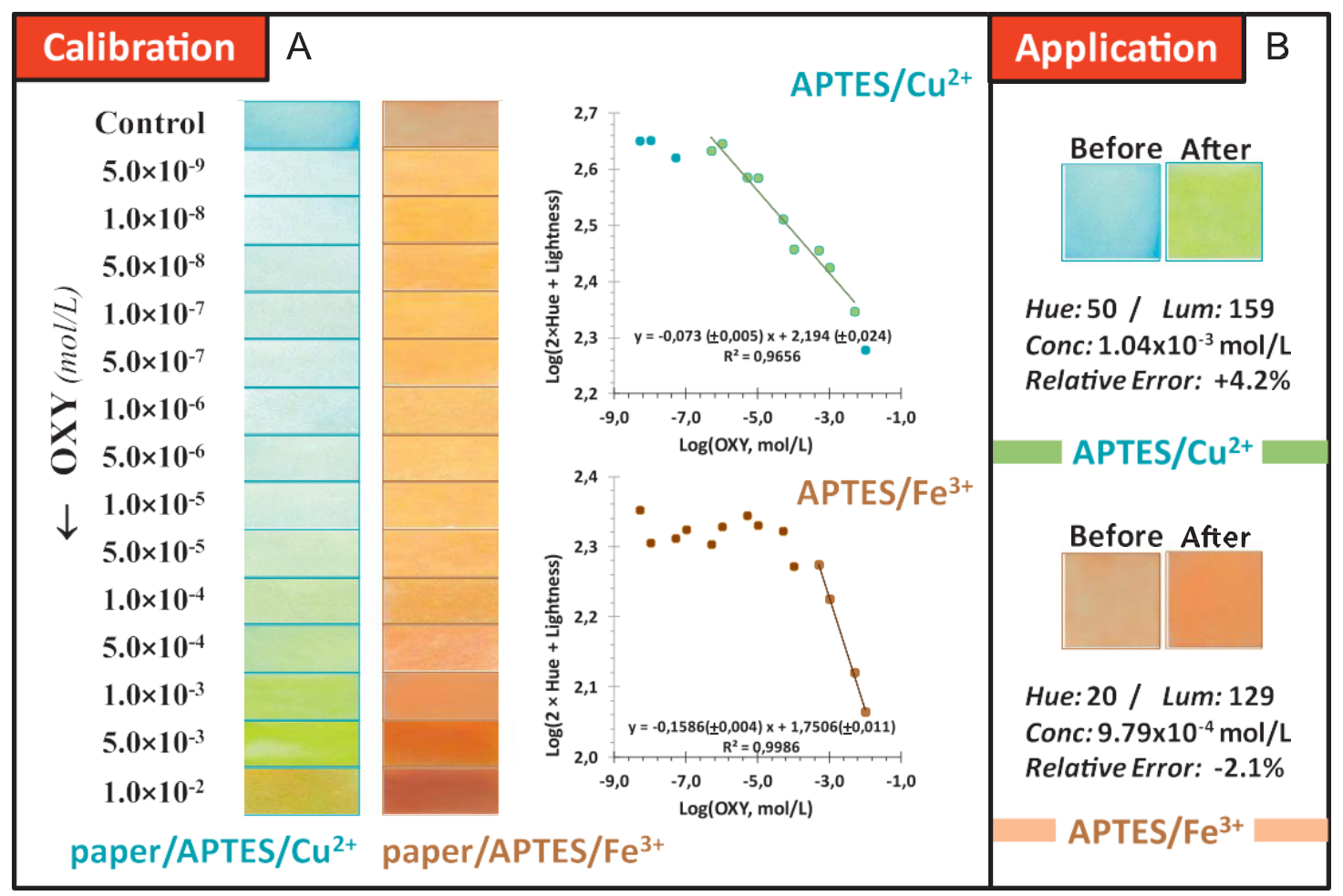

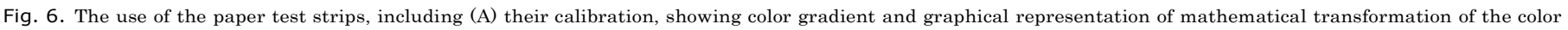
coordinates with the logarithm OXY concentration, and (B) their application. 
common reaction between OXY and TTC has eventually no practical effect on the final result because from the clinical point of view it makes no sense a combination of the same kind of drugs in therapy. On the other hand, it was possible to associate the sensor response as directed to the Tetracycline's group and not specifically to the OXY.

\subsection{Analytical features of the paper test-strip}

The analytical application of the paper test strip requires a previous calibration of these materials with different concentrations of OXY. The incubation of the paper test strips in OXY solutions of different concentration should generate a color gradient, thereby allowing immediate semi-quantitative determination of samples with unknown concentration by visual comparison. The calibration of the paper test strips was made by incubating the modified papers in OXY standard solutions, varying from $5 \times 10^{-9}$ to $1.0 \times 10^{-2} \mathrm{~mol} / \mathrm{L}$, for $5 \mathrm{~min}$. The resulting colors may be seen in Fig. $6 \mathrm{~A}$. In these, a color gradient was detected by visual and local inspection for concentrations higher then $1 \times 10^{-6}$ or $1 \times 10^{-4} \mathrm{~mol} / \mathrm{L}$, using paper/APTES/Cu or paper/APTES/Fe, respectively. This gradient assumed a more intense color with the increasing concentration of OXY, which as more perceptible in the paper/APTES/Cu materials.

Quantitative information may be also easily attained by mathematical handling of the color coordinates of the papers. In general, color coordinates may be obtained after taking the picture with a digital camera and treating the results with the PAINT program of Windows (currently all possible in a smartphone: picture and coordinate extraction). There are two color systems used currently in computers: RGB and HSL. Each system relies on a specific mathematic model, meaning that one system may apply better than the other. Moreover, considering that each coordinate has limited values (upper and maximum) and that the differences observed between the standards may involve different coordinates ( $\mathrm{R}, \mathrm{G}$ and/or B; or H, S and/or L), with different intensities, a linear correlation of a calibration curve should reflect these changes and intensities. The mathematical manipulation of the color coordinates was tried next, by following a trial and error approach for HSL model. The condition leading to a linear correlation between the variables of interest used $\log (2 \times$ Hue $p$ Lightness $)$ against log (OXY concentration). The linear trends found for the paper/APTES/ $\mathrm{Cu}$ and paper/APTES/Fe materials are indicated in Fig. 6A, and corresponded to the concentration ranges $5 \times 10^{-7}-5 \times 10^{-3}$ and $5 \times 10^{-4}-1 \times 10^{-2} \mathrm{~mol} / \mathrm{L}$, respectively.

Overall, the color gradient of the paper/APTES/Cu materials was more intense, and observed for lower concentrations of OXY, enabling the production of quantitative data for OXY concentrations higher $5 \times 10^{-7} \mathrm{~mol} / \mathrm{L}$ (equivalent to $30.3 \mathrm{ng} / \mathrm{mL}$ ).

\subsection{Application}

The possible application of the paper test strips was made by spiking an environmental water ( $\mathrm{pH} \quad$ "' 6.5) with OXY $1.0 \times 10^{-3} \mathrm{~mol} / \mathrm{L}$. The results obtained may be seen in Fig. $6 \mathrm{~B}$. Visual inspection and comparison with the standards (Fig. 6A) allowed a semi-quantitative and accurate determination of the OXY amount in the water.

The quantitative determination was made by using the linear regression equations of the calibration graphs and the average color coordinates extracted for these samples (indicated in Fig. 6B). The results obtained with paper/APTES/Cu and paper/APTES/Fe materials were $1.04 \times 10^{-3}$ and $9.79 \times 10^{-4} \mathrm{~mol} / \mathrm{L}$, respectively, corresponding to relative errors of $\mathbf{p} 4.2 \%$ and $-2.1 \%$.

Overall, the application of the paper test-strips was found accurate and precise, being the results obtained simply by incubating a paper in the solution to be analyzed, just as in the universal $\mathrm{pH}$ paper.

\section{Conclusions}

A low cost and disposable paper test strip was developed for quick screening of OXY in aquaculture environment. It consisted on the chemical modification of the glucose moieties of the paper with APTEs and after with $\mathrm{Cu}$ (II) or $\mathrm{Fe}$ (III). The color changes observed on the surface of the material were almost immediate and allowed semi-quantitative determination after visual inspection and comparison to the color gradient of standard solutions (similar approach to that of the universal $\mathrm{pH}$ paper). The paper modified with copper was more sensitive to lower concentration than the paper assembled with iron. Capturing the images of the paper with a camera and extracting the color coordinates with the Paint program of windows allowed a quantitative approach and widened the range of concentrations to which it was applied, with substantial decreasing in the limit of detection. In general, the reaction taking place on the surface of the modified paper seems to be unaffected by other antibiotic drugs and other compounds, although it is expected to be extended to other Tetracycline drugs. The application of the paper to an environmental sample has produced accurate data, making this method an excellent approach to carry out field monitoring of Tetracycline's in aquaculture facilities.

Overall, the paper test strips proposed herein are inexpensive; allow quick, low-cost, simple, equipment-free and environmentalfriendly determinations; thereby being suitable for field applications. Considering that the overall paper modification took less than $1 \mathrm{~h}$ and the laboratory scale experiments accommodated the simultaneous production of 100 papers, the process also seems suitable to a mass-scale production.

\section{Acknowledgements}

HIASG acknowledges Industrial Laborum Ibérica, SA, for personal funding.

\section{References}

Brown, Doc Brown's Chemistry website $\langle$ http://www.docbrown.info/page07/transi tion06Fe.htm>, 2000-2013 (accessed 17.07.14).

Cabello, F.C., 2006. Heavy use of prophylactic antibiotics in aquaculture: a growing problem for human and animal health and for the environment. Environ. Microbiol. 8 (7), 1137-1144.

Colt, J., 2006. Water quality requirements for reuse systems. Aquac. Eng. 34 (3), $143-156$.

Decreto-Lei no. 236/98 de 1 de Agosto. Diário da República no. 176/98-I Série A. Ministério do Ambiente, Lisboa.

Directive 2006/7/EC of the European Parliament and of the Council of 15 February 2006, Concerning the management of bathing water quality and repealing Directive 76/160/EEC, Official Journal of the European Communities, L64, 2006, pp. 37-51.

European Commission, 2010. Directive 2000/60/CE of October 23th. Off. J. Eur. Comm., L327/1.

EEA, 2010. The European Environment - State and Outlook 2010: Synthesis. European Environment Agency, Copenhagen.

Kemper, N., 2008. Veterinary antibiotics in the aquatic and terrestrial environment. Ecol. Indic. 8 (1), 1-13.

Kuemmerer, K., 2009. Antibiotics in the aquatic environment - a review - Part I. Chemosphere 75 (4), 417-434.

Lara Guerreiro, J.R., Ferreira Sales, M.G., 2011. Disposable solid state probe for optical screening of chlorpromazine. Microchim. Acta 175 (3-4), 323-331.

Li, P., Li, X., Saravanan, R., Li, C.M., Leong, S.S.J., 2012. Antimicrobial macromolecules: synthesis methods and future applications. RSC Adv. 2 (10), 4031-4044.

Luis Martinez, J., 2009. Environmental pollution by antibiotics and by antibiotic resistance determinants. Environ. Pollut. 157 (11), 2893-2902. 
Sarmah, A.K., Meyer, M.T., Boxall, A.B.A., 2006. A global perspective on the use, sales, exposure pathways, occurrence, fate and effects of veterinary antibiotics (VAs) in the environment. Chemosphere 65 (5), 725-759.

Silva, T.I.B., Moreira, F.T.C., Truta, L.A.A.N.A., Sales, M.G.F., 2012. Novel optical PVC probes for on-site detection/determination of fluoroquinolones in a solid/liquid interface: application to the determination of Norfloxacin in aquaculture water. Biosens. Bioelectron. 36 (1), 199-206.
Szymanska-Chargot, M., Cybulska, J., Zdunek, A., 2011. Sensing the structural differences in cellulose from apple and bacterial cell wall materials by Raman and FT-IR spectroscopy. Sensors 11 (6), 5543-5560.

WHO, 2000. Overcoming Antibiotic Resistance, World Health Organization Report in Infectious Diseases. WHO, Geneva. 